VoL. 42 (1990) [21-31]

\title{
ITERATIVE SOLUTION OF NONLINEAR EQUATIONS OF THE MONOTONE TYPE IN BANACH SPACES
}

\section{C.E. Chidume}

Let $E$ be a real Banach space with a uniformly convex dual, and let $K$ be a nonempty closed convex and bounded subset of $E$. Suppose $T: K \rightarrow K$ is a continuous monotone map. Define $S: K \rightarrow K$ by $S x=f-T x$ for each $x$ in $K$ and define the sequence $\left\{x_{n}\right\}_{n=0}^{\infty}$ iteratively by $x_{0} \in K, x_{n+1}=\left(1-C_{n}\right) x_{n}+C_{n} S x_{n}$, $n \geqslant 0$, where $\left\{C_{n}\right\}_{n=0}^{\infty}$ is a real sequence satisfying appropriate conditions. Then, for any given $f$ in $K$, the sequence $\left\{x_{n}\right\}_{n=0}^{\infty}$ converges strongly to a solution of $\boldsymbol{x}+T \boldsymbol{x}=f$ in $K$. Explicit error estimates are also computed. A related result deals with iterative solution of nonlinear equations of the dissipative type.

\section{INTRODUCTION}

Let $E$ be an arbitrary real Banach space. A mapping $T$ with domain $D(T)$ and range $R(T)$ in $E$ is said to be monotone [10] if the inequality

$$
\|x-y\| \leqslant\|x-y+t(T x-T y)\|,
$$

holds for each $x, y$ in $D(T)$ and some $t>0$. If the inequality (1) holds for all $t>0$, then $T$ is called accretive [2]. The accretive operators were introduced independently by Browder [2] and Kato [10]. If $E=H$, a Hilbert space, one of the earliest problems in the theory of monotone operators was to solve the equation $x+T x=f$ for $x$, given an element $f$ of $H$ and a monotone operator $T$ (see, for example, $[4,6,7,9,14,15$, 23, 24]). In [2], Browder proved that if $T$ is locally Lipschitzian and accretive then $T$ is $m$-accretive, that is, $(I+T) E=E$, so that for any given $f \in E$, the equation $x+T x=f$ has a solution. This result was subsequently generalised by Martin [13] to the continuous accretive operators. Zarantonello [24] also proved that if $H$ is a Hilbert space and $T$ is a monotone and Lipschitzian mapping of $H$ into itself then $x+T x=f$ has a unique solution in $H$.

A class of operators closely related to the class of accretive operators is the class of dissipative operators. An operator $A$ is dissipative if and only if $(-A)$ is accretive and $A$ on $E$ is called $m$-dissipative if $(I-\lambda A) E=E$ for $\lambda>0$. Browder [2] proved the following theorem:

\section{Received 14 August 1989}

The author hereby expresses his gratitude to Professor Simeon Reich of the University of Southern California for sending him some of his reprints/preprints. Research supported by a grant from TWAS (Grant No. MP88-14), I.C.T.P., Trieste, Italy.

Copyright Clearance Centre, Inc. Serial-fee code: 0004-9729/90 \$A2.00+0.00. 
Theorem B. Let $A$ be a single-valued dissipative operator on $E$ which is locally Lipschitzian on $E=D(A)$. Then $A$ is m-dissipative.

Recently, Ray [16] gave an elementary proof of Theorem B by employing a fixed point theorem of Caristi, [5].

Iterative methods for approximating a solution (when one exists) of the equation

$$
x+T x=f
$$

have been studied by various authors. In [9], Dotson showed that if $T: H \rightarrow H$ is monotone and Lipschitzian with Lipschitz constant 1 (in this case $T$ is called nonexpansive in the terminology of [11]), an iteration process of the type introduced by Mann [12], under suitable conditions, converges strongly to the unique solution of equation (2). In [6], the author constructed an approximation method which converges strongly to a solution of the equation (2) where $T: K \rightarrow H$ is a monotone Lipschitzian operator with Lipschitz constant $L \geqslant 1$ and $K$ is a nonempty closed convex subset of $H$ and $T$ has a fixed point in $K$. The results of the author [6] thus generalise the above result of Dotson both in the domain of the operator $T$ and in the range of its Lipschitz constant. In [4], Bruck Jr., considered an iteration process, in Hilbert space, for approximating a solution of the equation $f \in x+T x$ where $T: H \rightarrow H$ is a multivalued monotone operator. He proved that if $D(T)$ is an open domain of $T$ in $H$ and $f \in R(I+T)$ then there exist a neighbourhood $N$ in $D(T)$ of $\bar{x}=(I+T)^{-1} f$ and a real number $\sigma_{1}>0$ such that for any $\sigma \geqslant \sigma_{1}$, any initial guess $x_{1} \in N$ and any single-valued section $T_{0}$ of $T$, the sequence generated from $x_{1}$ by $x_{n+1}=x_{n}-(n+\sigma)^{-1}\left(x_{n}+T_{0} x_{n}-f\right)$ remains in $D(T)$ and converges to $\bar{x}$ with estimate $\left\|x_{n}-\bar{x}\right\|=O\left(n^{-1 / 2}\right)$. No continuity assumption was made on the map $T$. This result has recently been extended by the author [7] to $L_{p}$ spaces for $2 \leqslant p<\infty$. The method used in [7] could not be modified to yield any convergence result for $L_{p}$ spaces when $1<p<2$. More recently, the author [8] again considered the equation (2) in $L_{p}$ spaces for $2 \leqslant p<\infty$ when $T$ is singlevalued. Suppose $E=L_{p}$ (or $\ell_{p}$ ), $p \geqslant 2$, and $K$ is a nonempty closed convex subset of $E$. Suppose $T: K \rightarrow K$ is a monotone Lipschitzian mapping with Lipschitz constant $L \geqslant 1$. Define the sequence $\left\{x_{n}\right\}_{n=0}^{\infty}$ by $x_{0} \in K, x_{n+1}=(1-\lambda) x_{n}+\lambda\left(f-T x_{n}\right)$ for $n \geqslant 0$, where $\lambda=\left[(p-1)(1+L)^{2}\right]^{-1}$. The author $[8]$ proved that $\left\{x_{n}\right\}_{n=0}^{\infty}$ converges strongly to a solution of equation (2). Moreover, convergence is at least as fast as a geometric progression with ratio $(1-\lambda)^{1 / 2}$. Unfortunately, no convergence theorem could be proved in [8] for $L_{p}$ spaces with $1<p<2$.

It is our purpose in this paper to prove convergence theorems for an iterative method for approximating a solution of the equation

$$
x+T x=f
$$


in real Banach spaces much more general than $L_{p}$ spaces for $2 \leqslant p<\infty$. In particular, our results will include all $L_{p}$ (and $\ell_{p}$ ) spaces for $1<p<\infty$. In fact, we shall prove that if $E$ is any real Banach space with a uniformly convex dual $E^{*}$, and if $T: K \rightarrow K$ is a continuous monotone map where $K$ is a nonempty closed convex and bounded subset of $E$, an iteration process of the Mann-type converges strongly to a solution of equation (3) for any starting point $x_{0}$ in $K$. We shall also prove some related convergence theorems for the iterative approximation of a solution of the equation

$$
x-\lambda A x=f, \quad \lambda>0,
$$

where $A$ is a Lipschitzian dissipative operator on $E$.

\section{Preliminaries}

Let $E$ be a real Banach space and $E^{*}$ its dual. We shall denote by $J$ the normalised duality mapping from $E$ to $2^{E^{*}}$ given by

$$
J x=\left\{f^{*} \in E^{*}:\left\|f^{*}\right\|^{2}=\|x\|^{2}=\left\langle x, f^{*}\right)\right\},
$$

where $\langle$,$\rangle denotes the generalised duality pairing. It is well known that if E^{*}$ is uniformly convex then $J$ is single-valued and is uniformly continuous on bounded sets (see for example, [22]). Thus, by a single-valued duality mapping we shall mean a mapping $j: E \rightarrow E^{*}$ such that for each $x \in E, j(x)$ is an element of $E^{*}$ which satisfies the following two conditions:

$$
\langle u, j(u)\rangle=\|j(u)\| \cdot\|u\|, \quad\|j(u)\|=\|u\| .
$$

In terms of the single-valued duality mapping $j$, a map $A$ with domain $D(A)$ and range $R(A)$ in a real Banach space $E$ is called monotone if

$$
\langle A x-A y, j(x-y)\rangle \geqslant 0,
$$

for all $x, y$ in $D(A)$, (see for example, [10]). In the sequel we shall need the following remarks:

REMARK 1. In [21, p.89], Reich proved that if $E^{*}$ is uniformly convex then there exists a continuous nondecreasing function

$$
b:[0, \infty) \rightarrow[0, \infty)
$$

such that

$$
b(o)=0, \quad b(c t) \leqslant c b(t) \text { for all } c \geqslant 1,
$$

and

$$
\|x+y\|^{2} \leqslant\|x\|^{2}+2\langle y, j(x)\rangle+\max \{\|x\|, 1\}\|y\| b(\|y\|),
$$

for all $x, y$ in $E$. 
REMARK 2. Nevanlinna and Reich [17] have shown that for any given continuous nondecreasing function $b(t)$ with $b(0)=0$, sequences $\left\{\lambda_{n}\right\}_{n=0}^{\infty}$ always exist satisfying:

(i) $0<\lambda_{n}<1$ for all $n \geqslant 0$;

(ii) $\sum_{n=0}^{\infty} \lambda_{n}=\infty$, and

(iii) $\sum_{n=0}^{\infty} \lambda_{n} b\left(\lambda_{n}\right)<\infty$.

If $E=L_{p}, 1<p<\infty$, we can choose any sequence $\left\{\lambda_{n}\right\}_{n=0}^{\infty}$ in $\ell^{0} \backslash \ell^{1}$ with $s=p$ if $1<p \leqslant 2$ and, $s=2$, if $p \geqslant 2$.

\section{Main Results}

We prove the following theorems:

THEOREM 1. Let $E$ be a real Banach space with a uniformly convex dual space, $E^{*}$, and let $K$ be a nonempty closed convex and bounded subset of $E$. Suppose $T: K \rightarrow K$ is a continuous monotone map. Define $S: K \rightarrow K$ by $S x=f-T x$ for each $x$ in $K$. Define the sequence $\left\{x_{n}\right\}_{n=0}^{\infty}$ iteratively by $x_{0} \in K$,

$$
x_{n+1}=\left(1-\lambda_{n}\right) x_{n}+\lambda_{n} S x_{n}
$$

for $n \geqslant 0$, where $\left\{\lambda_{n}\right\}_{n=0}^{\infty}$ is a real sequence satisfying:

(i) $0<\lambda_{n}<1$ for all $n \geqslant 0$;

(ii) $\sum_{n=0}^{\infty} \lambda_{n}=\infty$, and

(iii) $\sum_{n=0}^{\infty} \lambda_{n} b\left(\lambda_{n}\right)<\infty$.

Then, for any given $f \in K$, the sequence $\left\{x_{n}\right\}_{n=0}^{\infty}$ converges strongly to a solution of $x+T x=f$ in $K$.

PROOF: The existence of a solution of $x+T x=f$ follows from [13]. Let $q$ denote a solution of this equation. Observe that $q$ is a fixed point of $S$. Moreover, the montonicity of $T$ implies,

$$
\langle S x-S y, j(x-y)\rangle=-\langle T x-T y, j(x-y)\rangle \leqslant 0,
$$

for each $x, y$ in $K$. Furthermore, using equation (7) and inequalities (6) and (8), we 
obtain:

$$
\begin{aligned}
\left\|x_{n+1}-q\right\|^{2}= & \left\|\left(1-\lambda_{n}\right)\left(x_{n}-q\right)+\lambda_{n}\left(S x_{n}-S q\right)\right\|^{2} \\
\leqslant & \left(1-\lambda_{n}\right)^{2}\left\|x_{n}-q\right\|^{2}+2 \lambda_{n}\left(1-\lambda_{n}\right)\left(S x_{n}-S q, j\left(x_{n}-q\right)\right\rangle \\
& +\max \left\{\left(1-\lambda_{n}\right)\left\|x_{n}-q\right\|, 1\right\} \lambda_{n}\left\|S x_{n}-S q\right\| b\left(\lambda_{n}\left\|S x_{n}-S q\right\|\right) \\
\leqslant & \left(1-\lambda_{n}\right)^{2}\left\|x_{n}-q\right\|^{2} \\
& +\max \left\{\left(1-\lambda_{n}\right)\left\|x_{n}-q\right\|, 1\right\} \lambda_{n}\left\|S x_{n}-S q\right\| \max \left\{\left\|S x_{n}-S q\right\|, 1\right\} b\left(\lambda_{n}\right) \\
\leqslant & \left(1-\lambda_{n}\right)\left\|x_{n}-q\right\|^{2}+M \lambda_{n} b\left(\lambda_{n}\right), \text { for some } M>0,
\end{aligned}
$$

since $K$ is bounded. Hence we have:

$$
\left\|x_{n+1}-q\right\|^{2} \leqslant\left(1-\lambda_{n}\right)\left\|x_{n}-q\right\|^{2}+M \lambda_{n} b\left(\lambda_{n}\right) .
$$

Set $\rho_{n}=\left|x_{n}-q\right|^{2}$ and $M \lambda_{n} b\left(\lambda_{n}\right)=\sigma_{n}$. Then, inequality (9) becomes:

$$
\rho_{n+1} \leqslant\left(1-\lambda_{n}\right) \rho_{n}+\sigma_{n} .
$$

A simple induction on inequality (10) yields:

$$
0 \leqslant \rho_{n+1} \leqslant \prod_{j=1}^{n}\left(1-\lambda_{j}\right) \rho_{1}+\beta_{n+1}
$$

where

$$
\beta_{n+1}= \begin{cases}\sigma_{1}, & n=1 \\ \sigma_{n}+\sum_{i=1}^{n-1} \sigma_{i} \prod_{j=i+1}^{n}\left(1-\lambda_{j}\right), & n>1 .\end{cases}
$$

For any fixed integer $k$, with $1<k \leqslant n-1$, we obtain:

$$
\beta_{n+1}=\sigma_{n}+\sum_{i=1}^{k} \sigma_{i} \prod_{j=i+1}^{n}\left(1-\lambda_{j}\right)+\sum_{i=k+1}^{n-1} \sigma_{i} \prod_{j=i+1}^{n}\left(1-\lambda_{j}\right) .
$$

Since $\delta_{n} \in[0,1]$, the above equation yields,

$$
0 \leqslant \beta_{n+1} \leqslant\left(\sum_{i=1}^{k} \sigma_{i}\right) \prod_{j=k+1}^{n}\left(1-\lambda_{j}\right)+\sum_{i=k+1}^{n} \sigma_{i} .
$$

Condition (ii) implies $\lim _{n \rightarrow \infty} \prod_{j=k+1}^{n}\left(1-\lambda_{j}\right)=0, k \geqslant 1$, so that

$$
0 \leqslant \lim _{n \rightarrow \infty} \inf \beta_{n} \leqslant \lim _{n \rightarrow \infty} \sup \beta_{n} \leqslant \sum_{i=k+1}^{\infty} \sigma_{i}
$$


Since inequaltiy (12) holds for arbitrary $k \geqslant 1$, and since condition (iii) implies $\lim _{k \rightarrow \infty} \sum_{i=k+1}^{\infty} \sigma_{i}=0$, it follows that

$$
\lim _{n \rightarrow \infty} \inf \beta_{n}=\lim _{n \rightarrow \infty} \sup \beta_{n}=\lim _{n \rightarrow \infty} \beta_{n}=0
$$

From inequality (11) and equation (13) we obtain that $\rho_{n} \rightarrow 0$ as $n \rightarrow \infty$ so that $x_{n} \rightarrow q$ as $n \rightarrow \infty$.

Corollary 1. Let $E=L_{p}$ (or $\left.\ell_{p}\right), 1<p \leqslant 2$, and let $K, T$ and $S$ be as in Theorem 1. Define the sequence $\left\{x_{n}\right\}_{n=0}^{\infty}$ iteratively by $x_{0} \in K$,

$$
x_{n+1}=\left(1-\lambda_{n}\right) x_{n}+\lambda_{n} S x_{n}
$$

for $n \geqslant 0$, where $\left\{\lambda_{n}\right\}_{n=0}^{\infty}$ is a real sequence satisfying:

(i) $0<\lambda_{n}<1$ for all $n \geqslant 0$;

(ii) $\sum_{n=0}^{\infty} \lambda_{n}=\infty$; and

(iii) $\sum_{n=0}^{\infty} \lambda_{n}^{p}<\infty$.

Then for any given $f$ in $K$ the sequence $\left\{x_{n}\right\}_{n=0}^{\infty}$ converges strongly to a solution of $x+T x=f$ in $K$.

ProOF: The existence of a solution to $x+T x=f$ follows from [13]. Remark 2 and conditions (ii) and (iii) imply $\sum \lambda_{n} b\left(\lambda_{n}\right)<\infty$. The result follows from Theorem 1. $\square$

Corollary 2. Let $E=L_{p}$ (or $\left.\ell_{p}\right), 2 \leqslant p<\infty$, and let $K, T$ and $S$ be as in Theorem 1. Define the sequence $\left\{x_{n}\right\}_{n=0}^{\infty}$ iteratively by $x_{0} \in K$,

$$
x_{n+1}=\left(1-\lambda_{n}\right) x_{n}+\lambda_{n} S x_{n},
$$

for $n \geqslant 0$, where $\left\{\lambda_{n}\right\}_{n=0}^{\infty}$ is a real sequence satisfying:

(i) $0<\lambda_{n}<1$ for all $n \geqslant 0$,

(ii) $\sum_{n=0}^{\infty} \lambda_{n}=\infty$, and

(iii) $\sum_{n=0} \lambda_{n}^{2}<\infty$.

Then for any given $f \in K$, the sequence $\left\{x_{n}\right\}_{n=0}^{\infty}$ converges strongly to a solution of $x+T x=f$ in $K$.

PROOF: The proof follows exactly as in the proof of Corollary 1.

REMARK 3. The only use we have made of the continuity of $T$ in Theorem 1 and Corollaries 1 and 2 above is to obtain the existence of a solution to the equation $x+T x=f$. 
ERror ESTIMATES: For the error estimates we shall need the following definition and lemma:

Definition: The modulus of convexity of a real Banach space $E$ is the function

$$
\delta_{E}:[0,2] \rightarrow[0,1]
$$

defined by the following formula:

$$
\delta_{E}(\varepsilon)=\inf [1-\|(x+y) / 2\|: x, y \in E,\|x\|=1=\|y\|,\|x-y\| \geqslant \varepsilon]
$$

LEMMA. Reich, [18]. If $\delta_{E^{*}}(\varepsilon) \geqslant K \varepsilon^{r}$ for some $K>0$ and $r \geqslant 2$ then for $t \leqslant M$, $b(t) \leqslant c t^{s-1}$ with $s=r /(r-1)$. If $E=L_{p}, 1 \leqslant p<\infty$, then $s=p$ if $1<p \leqslant 2$; and $s=2$ if $2 \leqslant p<\infty$.

The above lemma enables us to obtain a convergence rate in the setting of Theorem 1. If we set $\lambda_{n}=s /(n+1)$ then $\left\|x_{n}-q\right\|=O\left(n^{(-(\bullet-1)) / 2}\right)$. To see this, observe that by the above lemma and inequality (9) we have:

$$
\left\|x_{n+1}-q\right\|^{2} \leqslant\left(1-\frac{s}{n+1}\right)\left\|x_{n}-q\right\|^{2}+M\left(\frac{s}{n+1}\right) c\left(\frac{s}{n+1}\right)^{d-1}
$$

so that

$$
\left\|x_{n+1}-q\right\|^{2} \leqslant\left(1-\frac{s}{n+1}\right)\left\|x_{n}-q\right\|^{2}+M^{*} \frac{1}{(n+1)^{2}}
$$

for some constant $M^{*}>0$. Let $M_{2}=\max \left\{\left\|x_{1}-q\right\|, M^{*}\right\}$. Then clearly, $\left\|x_{1}-q\right\| \leqslant M_{2}$

Claim:

$$
\left\|x_{n}-q\right\|^{2} \leqslant M_{2} \frac{1}{n^{s-1}} .
$$

Proof of this claim is by induction. For $n=1$, the claim follows from the definition of $M_{2}$. Assume (15) holds for $n=k$. Then from (14),

$$
\begin{aligned}
\left\|x_{k+1}-q\right\|^{2} & \leqslant\left(1-\frac{s}{k+1}\right)\left\|x_{k}-q\right\|^{2}+M^{*} \frac{1}{(k+1)^{2}} \\
& \leqslant\left(1-\frac{1}{k+1}\right)^{\prime} M_{2} \frac{1}{k^{s-1}}+M^{*} \frac{1}{(k+1)^{s}} \\
& \leqslant\left(1-\frac{1}{k+1}\right)^{\prime} M_{2} \frac{1}{k^{s-1}}+M_{2} \frac{1}{(k+1)^{2}} \\
& =M_{2} \frac{1}{(k+1)^{s-1}}
\end{aligned}
$$


and so, by induction, (15) holds for all positive integers, and establishes that

$$
\left\|x_{n}-q\right\|=0\left(n^{-(s-1) / 2}\right) .
$$

In particular, if $E=L_{p}, 1<p<\infty$, we have:

$$
\left\|x_{n}-q\right\|=0\left(n^{-(p-1) / 2}\right) \text { if } 1<p \leqslant 2
$$

and,

$$
\left\|x_{n}-q\right\|=0\left(n^{-1 / 2}\right) \quad \text { if } 2 \leqslant p<\infty .
$$

We now consider iterative methods for single-valued dissipative and Lipschitz maps, and prove the following theorem:

THEOREM 2. Let $E$ be a real Banach space with a uniformly convex dual space, $E^{*}$. Let $K$ be a nonempty closed convex bounded subset of $E$. Suppose $A: K \rightarrow K$ is a single-valued dissipative and Lipschitzian mapping of $K$ into itself with Lipschitz constant $L \geqslant 1$. Define $G: K \rightarrow K$ by $G x=\lambda A x+f$ for arbitrary $x \in K$ and fixed $f$ in $K$. Define the sequence $\left\{x_{n}\right\}_{n=0}^{\infty}$ iteratively by $x_{0} \in K$,

$$
x_{n+1}=\left(1-\lambda_{n}\right) x_{n}+\lambda_{n} G x_{n}, n \geqslant 0,
$$

where $\left\{\lambda_{n}\right\}_{n=0}^{\infty}$ is a real sequence satisfying:

(i) $0<\lambda_{n}<1$ for all $n \geqslant 0$,

(ii) $\sum_{n=0}^{\infty} \lambda_{n}=\infty$, and

(iii) $\sum_{n=0}^{\infty} \lambda_{n} b\left(\lambda_{n}\right)<\infty$.

Then $\left\{x_{n}\right\}_{n=0}^{\infty}$ converges strongly to a solution of the equation

$$
x-\lambda A x=f \text { in } K .
$$

Proof: The existence of a solution to equation (16) follows from Theorem $B$. Let $q$ denote a solution. Observe that $q$ is a fixed point of $G$. Moreover, since $A$ is dissipative we have

$$
\left\langle G x_{n}-G q, j\left(x_{n}-q\right)\right\rangle=\lambda\left\langle A x_{n}-A q, j\left(x_{n}-q\right)\right\rangle \leqslant 0 .
$$

So,

$$
\begin{aligned}
\left\|x_{n+1}-q\right\|^{2}= & \left\|\left(1-\lambda_{n}\right)\left(x_{n}-q\right)+\lambda_{n}\left(G x_{n}-G q\right)\right\|^{2} \\
\leqslant & \left(1-\lambda_{n}\right)^{2}\left\|x_{n}-q\right\|^{2}+2 \lambda_{n}\left(1-\lambda_{n}\right)\left\langle G x_{n}-G q, j\left(x_{n}-q\right)\right) \\
& +\max \left\{\left(1-\lambda_{n}\right)\left\|x_{n}-q\right\|, 1\right\} \lambda_{n}\left\|G x_{n}-G q\right\| b\left(\lambda_{n}\left\|G x_{n}-G q\right\|\right) \\
\leqslant & \left(1-\lambda_{n}\right)^{2}\left\|x_{n}-q\right\|^{2} \\
& +\max \left\{\left(1-\lambda_{n}\right)\left\|x_{n}-q\right\|, 1\right\} \lambda_{n}\left\|G x_{n}-G q\right\| \max \left\{\left\|G x_{n}-G q\right\|, 1\right\} b\left(\lambda_{n}\right) \\
\leqslant & \left(1-\lambda_{n}\right)\left\|x_{n}-q\right\|^{2}+M \lambda_{n} b\left(\lambda_{n}\right), \quad \text { for some constant } M>0 .
\end{aligned}
$$


Now, set $\rho_{n}=\left\|x_{n}-q\right\|^{2}$, so that the above inequality becomes

$$
\rho_{n+1} \leqslant\left(1-\lambda_{n}\right) \rho_{n}+\sigma_{n}
$$

which is exactly inequality (10). The rest of the argument now follows exactly as in the proof of Theorem 1 to yield that $x_{n} \rightarrow q$ as $n \rightarrow \infty$, completing the proof of Theorem 2.

Corollary 1. Let $E=L_{p}, 1<p \leqslant 2$ and let $K, A$ and $G$ be as in Theorem 2 . Define the sequence $\left\{x_{n}\right\}_{n=0}^{\infty}$ iteratively by $x_{0} \in K$,

$$
x_{n+1}=\left(1-\lambda_{n}\right) x_{n}+\lambda_{n} G x_{n}, \quad n \geqslant 0
$$

where $\left\{\lambda_{n}\right\}_{n=0}^{\infty}$ is a real sequence satisfying:

(i) $0<\lambda_{n}<1$, for all $n$,

(ii) $\sum_{n=0}^{\infty} \lambda_{n}=\infty$, and

(iii) $\sum_{n=0}^{\infty} \lambda_{n}^{p}<\infty$.

Then $\left\{x_{n}\right\}_{n=0}^{\infty}$ converges strongly to a solution of the equation $x-\lambda A x=f$ in $K$.

Proof: Observe that conditions (ii) and (iii) imply that $\left\{\lambda_{n}\right\}_{n=0}^{\infty} \in \ell^{p} \backslash \ell^{i}$ and so by Remark $2, \sum_{n=0}^{\infty} \lambda_{n} b\left(\lambda_{n}\right)<\infty$. Corollary 1 then follows from Theorem 2.

Corollary 2. Let $E=L_{p}, 2 \leqslant p<\infty$, and let $K, A$ and $G$ be as in Theorem 2. Define the sequence $\left\{x_{n}\right\}_{n=0}^{\infty}$ iteratively by $x_{0} \in K$,

$$
x_{n+1}=\left(1-\lambda_{n}\right) x_{n}+\lambda_{n} G x_{n}, \quad n \geqslant 0,
$$

where $\left\{\lambda_{n}\right\}_{n=0}^{\infty}$ is a real sequence satisfying:

(i) $0<\lambda_{n}<1$ for all $n$,

(ii) $\sum_{n=0}^{\infty} \lambda_{n}=\infty$, and

(iii) $\sum_{n=0}^{\infty} \lambda_{n}^{2}<\infty$.

Then $\left\{x_{n}\right\}_{n=0}^{\infty}$ converges strongly to a solution of the equation $x-\lambda A x=f$ in $K$.

Proof: Since $\left\{\lambda_{n}\right\}_{n=0}^{\infty} \in \ell^{2} \backslash \ell^{1}$, Remark 2 implies $\sum_{n=0}^{\infty} \lambda_{n} b\left(\lambda_{n}\right)<\infty$, and the Corollary follows from Theorem 2.

Remark 4. The error estimates for Theorem 2 and Corollaries 1 and 2 of this theorem are obtained as in Theorem 1 (and Corollaries 1 and 2 of Theorem 1). 
REMARK 5. Corollaries 1 and 2 of Theorem 1 generalise the main result of Dotson [9] and of the author [6] in several ways. In both [9] and [6] convergence theorems were proved in Hilbert spaces and the operator $T$ was assumed to be monotone and Lipschitzian. In Corollaries 1 and 2 of Theorem 1 above, convergence of the iteration scheme is proved for the much larger class of Banach spaces $L_{p}$ spaces, $1<p<\infty$, and also for the much larger class of monotone continuous maps. Moreover, the rate of convergence established in Corollaries 1 and 2 of Theorem 1 above agrees with that established in both [6] and [9].

REMARK 6. It is a consequence of the proof of Theorem 1 that, under the hypotheses of the theorem, the solution of the given equation must be unique. The element $q \in F(S)$, where $F(S)$ denotes the set of fixed points of $S$, was arbitrarily chosen. Suppose now there is a $q^{*} \in F(S)$ with $q^{*} \neq q$. Repeating the argument of the theorem relative to $q^{*}$, one sees that the sequence $\left\{x_{n}\right\}_{n=0}^{\infty}$ converges to both $q$ and $q^{*}$, showing that $F(S)=\{q\}$.

A similar argument shows that the solution of the equation in Theorem 2 is unique.

\section{REFERENCES}

[1] F.E. Browder, 'Nonlinear elliptic boundary value problems', Bull. Amer. Math. Soc. 69 (1967), 862-874.

[2] F.E Browder, 'Nonlinear mappings of nonexpansive and accretive type in Banach spaces', Bull. Amer. Math. Soc. 73 (1967), 875-882.

[3] F.E. Browder, 'Nonlinear monotone and accretive operators in Banach spaces', Proc. Nat. Acad. Sci. U.S.A. 61 (1968), 388-393.

[4] R.E. Bruck Jr., 'The iterative solution of the equation $y \in x+T x$ for a monotone operator $T$ in Hilbert space', Bull. Amer. Math. Soc. 79 (1973), 1258-1262.

[5] J. Caristi, 'Fixed point theorems for mappings satisfying inwardness conditions', Trans. Amer. Math. Soc. 215 (1976), 241-251.

[6] C.E. Chidume, 'An approximation method for monotone Lipschitzian operators in Hilbert space', J. Austral. Math. Soc. (series A) 41 (1986), 59-63.

[7] C.E. Chidume, 'The iterative solution of the equation $f \in x+T x$ for a monotone operator $T$ in $L_{p}$ spaces', J. Math. Anal. Appl. 116 (1986), 531-537.

[8] C.E. Chidume, 'Iterative solution of nonlinear equations of the monotone and dissipative types', Appl. Anal. 33 (1989), 79-86.

[9] W.G. Dotson, 'An iterative process for nonlinear monotonic nonexpansive operators in Hilbert space', Math. Comp. 32 (1978), 223-225.

[10] T. Kato, 'Nonlinear semigroups and evolution equations', J. Math. Soc. Japan 19 (1967), 508-520.

[11] W.A. Kirk, 'A fixed point theorem for mappings which do not increase distance', Amer. Math. Monthly 72 (1965), 1004-1006. 
[12] W.R. Mann, 'Mean value methods in iteration', Proc. Amer. Math. Soc. 4 (1953), 506-510.

[13] R.H. Martin Jr., 'A global existence theorem for autonomous differential equations in Banach spaces', Proc. Amer. Math. Soc. 26 (1970), 307-314.

[14] G.J. Minty, 'Monotone (nonlinear) operators in Hilbert spaces', Duke Math. J. 29 (1962), 541-546.

[15] W.V. Petryshyn, 'On the extension and solution of nonlinear operator equations', Ilinois J. Math. 10 (1966), 255-274.

[16] W.O. Ray, 'An elementary proof of surjectivity for a class of accretive operators', Proc. Amer. Math. Soc. 75 (1979), 255-258.

[17] O. Nevanlinna and S. Reich, 'Strong convergence of contraction semigroups and of iterative methods for accretive operators in Banach spaces', Israel J. Math. 32 (1979), 44-58.

[18] S. Reich, 'Constructive techniques for accretive and monotone operators', Applied Nonlinear Analysis (1979), 335-345.

[19] S. Reich, 'Constructing zeros of accretive operators II', Applicable Analysis 9 (1979), 159-163.

[20] S. Reich, 'Strong convergence theorems for resolvents of accretive operators in Banach spaces', J. Math. Anal. Appl. 75 (1980), 287-292.

[21] S. Reich, 'An iterative procedure for constructing zeros of accretive sets in Banach spaces', Nonlinear Anal. 2 (1978), 85-92.

[22] V.L. Smul'Yan, 'On the derivation of the norm in a Banach space', Dokl. Akad. Nauk SSSR 27 (1940), 255-258.

[23] M.M. Vainberg, 'On the convergence of the method of steepest descent for nonlinear equations', Sibirsk. Math. Zh. Z.2 (1961), 201-220.

[24] E.H. Zarantonello, 'Solving functional equations by contractive averaging', in Technical Report No.160 (U.S. Army Math. Res. Centre, Madison, Wisconsin, 1960). 